\title{
Musculoskeletal Brucellosis in Adults in the United Arab Emirates: A Retrospective Study
}

\author{
Authors: \\ *Shamma Al Nokhatha,, Fatima AlKindi, ${ }^{1}$ Shaima Al Yassi,, Rayhan \\ Hashmey ${ }^{3}$ \\ 1. Division of Rheumatology, Tawam Hospital, Al Ain, United Arab Emirates \\ 2. Division of General Internal Medicine, Tawam Hospital, Al Ain, United Arab Emirates \\ 3. Division of Infectious Diseases, Tawam Hospital, Al Ain, United Arab Emirates \\ *Correspondence to shamma.alnokhatha@gmail.com \\ Disclosure: $\quad$ The authors have declared no conflicts of interest. \\ Received: \\ 22.11.20 \\ Accepted: \\ 15.01.21 \\ Keywords: \\ Arthritis, brucellosis, musculoskeletal (MSK) involvement, sacroiliac joint (SIJ), United \\ Arab Emirates (UAE). \\ Citation: \\ EMJ. 2021; DOI/10.33590/emj/20-00271
}

\section{Abstract}

Introduction: Brucellosis is a zoonotic infection caused by the aerobic Gram-negative bacteria coccobacilli, and is considered a public health problem in the Mediterranean region and Arabian Peninsula. This paper studied the clinical characteristics of musculoskeletal brucellosis and the outcomes of treatment in Al Ain City, United Arab Emirates.

Method: A retrospective chart review study was conducted at Tawam Hospital over seven years: January 2009-January 2016. Risk factors for brucellosis, musculoskeletal (MSK) manifestations, duration of Brucella infection (acute, subacute, chronic), and treatment were studied.

Results: A total of 99 patients were diagnosed with brucellosis during the study period; the mean age was 44 years, the majority were males (71\%), and the male to female ratio was 3:1. The most common risk factor for Brucella infection in the cohort was drinking raw milk (43.4\%). Fever was the most common presenting symptoms (93\%), followed by arthralgia, fatigue, and loss of appetite in 35,21 , and $14 \%$, respectively. The clinical manifestations of brucellosis in the cohort were MSK involvement (30\%), hepatitis (17\%), epididymo-orchitis (2\%), and endocarditis (1\%). Thirty percent of patients $(n=30)$ had MSK-specific symptoms and only one-third $(n=10)$ had confirmatory positive radiographic findings. The majority of patients had lumbar and sacroiliac joint involvement. Most of the patients received antibiotics for a 4-8-week duration and the overall relapse rate of Brucella infection was $10 \%$.

Conclusion: This study demonstrates that MSK involvement is a common manifestation in brucellosis, occurring in one-third of the cases. The index of suspicion should be high in brucellosis-endemic countries for early recognition and treatment.

\section{INTRODUCTION}

Brucellosis is a zoonotic infection, historically known as Maltese fever, caused by aerobic Gram-

negative coccobacilli, which was first identified by Sir David Bruce in 1887. ' Worldwide, the true prevalence of brucellosis is unknown; however, it is considered a public health problem in the 
Mediterranean region, Arabian Peninsula, India, Mexico, and part of the USA. Pappas et al. ${ }^{2}$ estimated the global incidence of brucellosis to be more than half a million cases annually, and the incidence in endemic disease areas to be from $<0.01->200$ cases per 100,000 of the population. In the United Arab Emirates (UAE), the total brucellosis notification rate between 2010 and 2015 was estimated to be 3.3 per 100,000 people per year in Abu Dhabi city, the capital of the UAE. ${ }^{3}$

Brucella is a facultative intracellular bacterium that infects animals and has various different species: Brucella melitensis (goats, sheep, camels), B. abortus (bovine, cattle, camels), B. suis (swine, cattle), B. canis (dogs), B. ovis (ram, sheep), and $B$. neotomae (desert rats). ${ }^{1}$ An epidemiological study measuring the prevalence of Brucella in 6,126 livestock in Abu Dhabi reported higher prevalence in sheep and goats (8.4\%) compared to camels (4.4\%). ${ }^{4}$ Risk factors of Brucella infection include drinking unpasteurised animal milk products, direct contact with infected animals, inhalation of aerosolised particles, and occupational and work-related diseases (shepherds, abattoir workers, veterinarians, dairy-industry professionals, and microbiologic laboratory workers) ${ }^{1,5,6}$ Clinical manifestations of Brucella infection vary depending on the organ involved. Common presenting symptoms are fever (78\%), chills (45\%), sweats (54\%), fatigue (39\%), weight loss (26\%), arthralgia (65\%), and abdominal pain (19\%) due to hepatomegaly or splenomegaly. ${ }^{7}$ Brucella infection is classified based on the time of clinical presentation as acute brucellosis ( $0-2$ months), subacute brucellosis (2-12 months), and chronic brucellosis ( $>12$ months). The laboratory findings might reveal leukocytosis (9\%), leukopenia (10\%), anaemia (40\%), thrombocytopenia (10\%), elevated liver enzymes (24\%), and inflammatory markers (>50\%; C-reactive protein [CRP], and erythrocyte sedimentation rate $[E S R])$. The majority of patients (94\%) will have a positive Brucella titre (tube agglutination test) and bacteraemia may be encountered in approximately $11 \%$ of cases. ${ }^{5}$

Musculoskeletal (MSK) involvement of brucellosis represents $10-85 \%$ of reported clinical manifestations such as peripheral arthritis, bursitis, sacroiliitis, osteomyelitis, spondylitis, and paraspinal abscess.,9 Spinal brucellosis is common in the elderly and predominately affects the L4-L5 spinal segment of the lumbar spine, followed by thoracic and cervical spine.,9,10 Diagnosis of osteoarticular brucellosis requires laboratory tests to confirm Brucella infection and imaging studies. MRI produces a high yield of images that detect radiological features of spinal brucellosis. There is no prior study, to the authors knowledge, describing the clinical manifestations of MSK brucellosis in the UAE; therefore, a retrospective study was conducted, with the aim of identifying the clinical characteristics of MSK brucellosis and outcomes of treatment in Al Ain, UAE.

\section{METHODS}

A retrospective chart review study was conducted at Tawam hospital (tertiary hospital, Al Ain, UAE) over seven years (January 2009January 2016). Ethical approval was obtained from Tawam Human Research Ethics Committee (T-HREC). Adult patients ( $>16$ years of age) diagnosed with brucellosis during the study period were involved. The diagnosis of brucellosis was confirmed by either a positive Brucella titre of >1:160 and/or positive blood culture. Citizens were described as 'nationals' and non-citizens as 'non-nationals'.

Demographic, clinical, and laboratory data were collected and analyzed using descriptive analysis. Risk factors for Brucella infection and clinical presenting symptoms were studied. Patients were classified into subgroups according to arthritis types, either peripheral or axial. Brucella infection was classified based on the time of clinical presentation as acute brucellosis ( $0-2$ months), subacute brucellosis (2-12 months), and chronic brucellosis (>12 months). ${ }^{5}$ Relevant blood tests were included, and confirmatory radiographic imaging was obtained in all patients with MSK complaints (plain radiograph/X-ray, CT scan, or MRI). Treatment of MSK brucellosis and outcomes were identified.

\section{RESULTS}

A total of 99 patients were diagnosed with brucellosis during the study period. The mean age was 44 years, the majority were males (71\%), and the male to female ratio was nearly 3:1. Twothirds of patients were nationals (63 out of 99; 
63.3\%). The most common factor for Brucella infection in the cohort was drinking raw milk (43 out of $99 ; 43.4 \%)$; the remaining are summarised in Table 1.

Fever was the most common symptom and accounted for $93 \%$ of all patients, followed by arthralgia, fatigue, and loss of appetite in 35, 21 , and $14 \%$, respectively. Brucella infection was confirmed in all patients based on elevated Brucella titres (B. melitensis and B. abortus) of $>1: 160$. Brucella bacteraemia (positive blood culture) was identified in only 57 patients. Other laboratory investigations revealed leukocytosis (white blood cell count $>11 \times 10^{9} / \mathrm{L}$ in 10 out of 99 ;
$10.1 \%$ ), anaemia (30 out of $99 ; 30.3 \%$ ), abnormal liver enzymes (47 out of 99; 47.5\%), and elevated CRP ( 83 out of $99 ; 83.8 \%$ ). Systemic complications of brucellosis in the cohort were MSK involvement (30\%), hepatitis (17\%), epididymo-orchitis (2\%), and endocarditis (1\%).

Of the patients with MSK involvement $(n=30)$, only one-third $(n=10)$ had confirmatory positive radiographic findings. The most common joint involved was the lumbar spine, accounting for $34.5 \% \quad(n=10)$ of the cases; followed by sacroiliac and shoulder joints, each accounting for $23.3 \%(n=7)$; and then the hip and knee joints, accounting for $13.3 \%(n=4)$.

Table 1: Demographic of patients $(n=99)$, risk factors for Brucella infection and the presenting symptoms.

\begin{tabular}{|c|c|}
\hline & $N=99$ \\
\hline \multicolumn{2}{|l|}{ Demographics } \\
\hline Male & 71 \\
\hline Female & 28 \\
\hline \multicolumn{2}{|l|}{ Risk factors } \\
\hline Farm contact with animals & 18 \\
\hline Raw milk drinking & 43 \\
\hline Boiled milk drinking & 3 \\
\hline Fresh cheese ingestion & 1 \\
\hline Ingestion of raw meatballs & 1 \\
\hline Laboratory worker & 0 \\
\hline Unknown transmission & 33 \\
\hline \multicolumn{2}{|l|}{ Symptoms } \\
\hline Arthralgia & 35 \\
\hline Fatigue & 21 \\
\hline Back pain & 23 \\
\hline Fever & 94 \\
\hline Sweating & 15 \\
\hline Headache & 10 \\
\hline Weight loss & 10 \\
\hline Loss of appetite & 15 \\
\hline Nausea & 3 \\
\hline Vomiting, abdominal pain & 10 \\
\hline Scrotal pain and swelling & 3 \\
\hline
\end{tabular}


The radiographic imaging using $\mathrm{X}$-ray and MRI were positive in 10 patients with MSK brucellosis (9 males, 1 female). Five patients had Brucella bacteraemia and all had high Brucella titres, ranging from $1: 320$ to $1: 10,240$. The majority of patients had lumbar and sacroiliac joint involvement, with radiographic imaging findings such as disc involvement, vertebral body destruction or abscess, unilateral sacroiliitis, and osteomyelitis. Two patients had septic arthritis related to Brucella infection involving the hip and knee joints, with positive radiological findings of joint effusion. The majority of patients with MSK brucellosis who had positive radiographic changes were diagnosed during the acute phase (90\%; n=9) (Table 2, Figure 1).

Details of brucellosis treatment were available in 88 out of 99 patients. All patients received combination therapy with at least two antibiotics. A third agent, mostly an aminoglycoside, was added to treatment in 27 patients (30.6\%) for MSK involvement. The most common antibiotics used were doxycycline (87 out of $88 ; 98.8 \%$ ), rifampin ( 76 out of $88 ; 86.3 \%$ ), gentamicin (20 out of $88 ; 22.7 \%$ ), trimethoprim/sulfamethoxazole (14 out of $88 ; 15.9 \%$ ), and ciprofloxacin (5 out of 88 ; $5.6 \%)$. The duration of therapy varied depending on severity and organ involvement. A majority of patients received 4-6 weeks of antibiotics (64 out of 88), followed by 8 weeks of therapy in 15 patients; only two patients required prolonged antibiotic treatment of more than 8 weeks. Surgical therapy was provided for patients $(n=2)$ with MSK brucellosis for draining vertebral abscess, septic arthritis, and discitis. The overall relapse rate of Brucella infection in the cohort $(n=99)$ was $10 \%$.

\section{DISCUSSION}

The burden of Brucella infection and its multisystem involvement is under-reported in some endemic areas. In a meta-analysis of the clinical manifestations of human brucellosis, adult males were affected in $56 \%$ of studies, and MSK complaints were common presenting symptoms (65\% arthralgia, 47\% myalgia, and 45\% back pain). The potential risk factors identified for brucellosis were consumption of unpasteurised dairy products in $64 \%$ of the studies, followed by contact with livestock in $42 \%$, occupational exposure in $6 \%$, and positive family history of brucellosis in 20\% (ranging from $17 \%$ to $46 \%$ ). Interestingly, some studies advocated for Brucella screening among families where one member had been diagnosed, due to the possibility of sharing the same risk factors. $5,7,11$

This study reports 99 cases of confirmed brucellosis; the mean age of patients was 44 years, and men were three times more affected than women. This demonstrates an increased risk to those men involved in animal husbandry. The most significant risk factor was consumption of unpasteurised milk. These findings are consistent with a study conducted in the same region, in which the disease affected mostly men with a mean age of 40 years and where the risk factors constituted of consumption of unpasteurised milk and dairy products. ${ }^{3}$ This may explain the importance of cultural and environmental impact.

The presenting symptoms of Brucella infection are nonspecific, and many cases were identified during work-up for fever of unknown origin. Differential diagnosis often includes other infectious and non-infectious chronic inflammatory conditions. Pourbagher et al. ${ }^{10}$ noted that fever, fatigue, loss of appetite, nausea, and diarrhoea were the main reported symptoms in the acute phase, while weight loss and palpitation were observed during the subacute phase and the osteoarticular manifestation during the chronic stage. ${ }^{10}$ Similarly, in this study, fever was the most common presenting symptom in $93 \%$ of patients, followed by arthralgia, fatigue, and loss of appetite in 35,21 , and $14 \%$, respectively. In contrast, osteoarticular involvements in this cohort were diagnosed during the acute phase. Physician education, a higher level of suspicion, and easy availability of serological testing and positive blood cultures may account for this finding.

In this cohort, 30 adult patients were identified with MSK-specific symptoms; one-third had confirmatory positive radiographic findings as described earlier. The reported MSK involvement in different studies varied; along with variation at the affected site, rheumatic complaints were reported in 20-85\% of brucellosis cases. ${ }^{12-14}$ In a study conducted in Turkey with 251 patients, $45.4 \%(n=114)$ of cases reported osteoarticular involvement, with the most common site being the sacroiliac joint (28.3\%) followed by lumbar spine $(10.4 \%))^{10}$ 
Table 2: Clinical characteristics of patients with musculoskeletal brucellosis and positive radiological findings.

\begin{tabular}{|c|c|c|c|c|c|c|c|}
\hline & Age & Gender & Stage & $\begin{array}{l}\text { Blood } \\
\text { culture }\end{array}$ & Brucella titre* & Complications & Radiographic changes \\
\hline Case 1 & 33 & Male & Acute & Negative & $1: 640$ & \begin{tabular}{|l|} 
Right \\
sacroiliitis
\end{tabular} & $\begin{array}{l}\text { X-ray SIJ: no abnormalities } \\
\text { MRI SIJ: unilateral right sacroiliitis }\end{array}$ \\
\hline Case 2 & 89 & Male & Acute & Positive & 1:10,240 & $\begin{array}{l}\text { Lumbar } \\
\text { abscess } \\
\text { collection } \\
\text { Hepatitis }\end{array}$ & $\begin{array}{l}\text { X-ray lumbar spine: intervertebral } \\
\text { space narrowing L1-L2, L3-L4, } \\
\text { and L5-S1 } \\
\text { MRI lumbar spine: collection in } \\
\text { the lumbar spine }\end{array}$ \\
\hline Case 3 & 80 & Male & Acute & Negative & $1: 1,280$ & \begin{tabular}{|l|} 
Lumbar \\
discitis and \\
osteomyelitis
\end{tabular} & $\begin{array}{l}\text { X-ray lumbar spine: vertebral } \\
\text { body destruction L4/5 } \\
\text { MRI lumbar spine: L4/L5 disc } \\
\text { and endplates signal abnormality, } \\
\text { highly suspicious of discitis and } \\
\text { osteomyelitis }\end{array}$ \\
\hline Case 4 & 35 & Male $^{+}$ & Acute & Negative & $1: 2,560$ & $\begin{array}{l}\text { Left knee } \\
\text { monoarthritis } \\
\text { (septic) }\end{array}$ & $\begin{array}{l}\text { X-ray left knee: no abnormalities } \\
\text { MRI left knee: effusion }\end{array}$ \\
\hline Case 5 & 20 & Male & Acute & Positive & $1: 2,560$ & \begin{tabular}{|l|} 
Septic \\
right-sided \\
sacroiliitis
\end{tabular} & $\begin{array}{l}\text { X-ray SIJ: no abnormalities } \\
\text { MRI SIJ: mild right-sided septic } \\
\text { arthritis with small collection } \\
\text { underneath the anterior } \\
\text { sacroiliac ligament }\end{array}$ \\
\hline Case 6 & 35 & Male & Acute & Positive & $1: 2,560$ & $\begin{array}{l}\text { Lumbar disc } \\
\text { involvement } \\
\text { Hepatitis }\end{array}$ & $\begin{array}{l}\text { X-ray lumbar spine: disc } \\
\text { involvement } \\
\text { MRI lumbar spine: disc } \\
\text { involvement L5/S1, nerve } \\
\text { compression }\end{array}$ \\
\hline Case 7 & 28 & Female & Acute & Negative & $1: 640$ & \begin{tabular}{|l} 
Left hip \\
monoarthritis \\
(septic) \\
Osteomyelitis
\end{tabular} & $\begin{array}{l}\text { X-ray hip: no abnormalities } \\
\text { MRI left hip: joint effusion }\end{array}$ \\
\hline Case 8 & 18 & Male & Acute & Positive & $1: 10,240$ & \begin{tabular}{|l|} 
Septic \\
left-sided \\
sacroiliitis
\end{tabular} & $\begin{array}{l}\text { X-ray SIJ: no abnormalities } \\
\text { MRI SIJ: left sacroiliitis }\end{array}$ \\
\hline Case 9 & 71 & Male & Subacute & Negative & $1: 640$ & $\begin{array}{l}\text { Lumbar discitis } \\
\text { Hepatitis }\end{array}$ & $\begin{array}{l}\text { X-ray lumbar spine: no } \\
\text { abnormalities } \\
\text { MRI lumbar spine: disc } \\
\text { involvement, discitis D1/L1 and } \\
\text { L3/L4 }\end{array}$ \\
\hline Case 10 & 31 & Male & Acute & Positive & $1: 320$ & $\begin{array}{l}\text { Sacroiliac } \\
\text { sclerosis }\end{array}$ & X-ray SIJ: left SIJ sclerosis \\
\hline
\end{tabular}

*both (Brucella abortus and B. melitensis).

texpatriate.

SIJ: sacroiliac joint. 


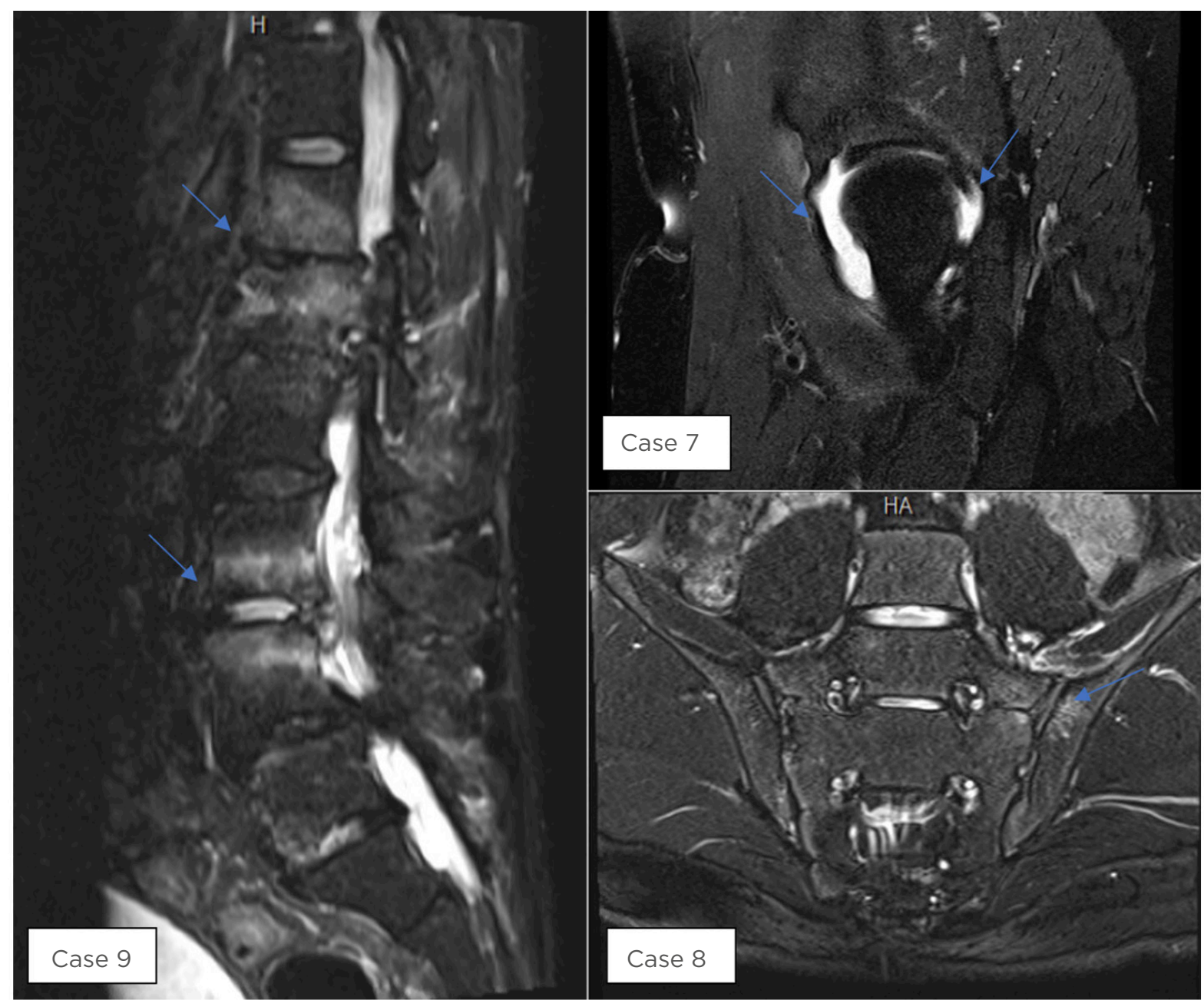

Figure 1: Positive MRI findings in selected cases as described in Table 2.

In another study from Saudi Arabia, with 84 cases of brucellosis, MSK complication was the most common symptom and $64 \%$ were found to have either peripheral arthritis, sacroiliitis, or spondylitis. ${ }^{15}$ In this study, almost $80 \%(n=8)$ of patients had axial involvement affecting lumbar and sacroiliac joints with positive radiographic imaging (disc involvement, discitis, vertebral body destruction, unilateral sacroiliitis). Peripheral involvement is less common compared with axial involvement. Furthermore, Buzgan et al. ${ }^{5}$ reported osteoarticular Brucella involvement in 260 cases out of 1,028 patients (25.3\%), involving peripheral arthritis (56.5\%), sacroiliitis (24.6\%) (unilateral in 51 cases), spondylitis (12.3\%), and paraspinal abscess (3.5\%). They received various therapeutic regimens for a duration of approximately 6-12 weeks and the overall relapse rate was $4.7 \%$ (in osteoarticular involvement: $8.5 \%){ }^{5}$
The imaging modalities used to diagnose MSK Brucella involvement varies in published literature. For peripheral arthritis, ultrasound can detect effusion. In one study, 5.2\% of patients presenting with arthralgia, diagnosed with bursitis ( 13 out of 251 Brucella-infected cases) using ultrasound, had negative synovial fluid culture. ${ }^{10}$ Brucellosis with sacroiliac joint involvement can be diagnosed using a plain radiograph (late findings), radionuclide bone scintigraphy (bone scan), or MRI. It is estimated that two-thirds of cases will have unilateral sacroiliitis and only one-third will be bilateral. A bone scan showed increased uptake in the sacroiliac joints. 5, 9, 10 Radiographs in vertebral brucellosis may reveal abnormalities in vertebral endplates, associated with irregularities and narrowing intervertebral disk spaces in subacute and chronic Brucella infection. MRI is very sensitive for detecting spondylodiscitis, vertebral destruction, spinal stenosis/abscess, 
and osteomyelitis. It is fundamental to rule out tuberculosis infection, which has predilection to same articular involvement (Pott's Disease) with chest radiographs, QuantiFERON tests, and acidfast bacilli stain and culture on biopsy.

The aim of medical therapy in cases of brucellosis is to prevent complications and relapse and to control the acute illness. Monotherapy and short duration of $<4$ weeks are not recommended in the treatment of brucellosis due to high risk of treatment failure and relapse. In a metaanalysis, the combination of streptomycin for 2-3 weeks plus doxycycline for 6 weeks had a lower failure rate than doxycycline plus rifampicin for 6 weeks. ${ }^{16,17}$ Other antibiotics, such as quinolones (e.g. ciprofloxacin and ofloxacin), aminoglycosides (e.g. gentamicin), and trimethoprim/sulfamethoxazole, have been used along with other combinations and have shown variable effects. ${ }^{8,18}$ The optimal duration of therapy for MSK brucellosis is not well established, but prolonged therapy is required for complicated cases. In the current cohort, all patients were on a combination of two antibiotics (doxycycline plus rifampicin regimen being the most common) and patients with MSK involvement required the addition of aminoglycosides (gentamicin). The majority of the patients received antibiotics for a duration of 4-8 weeks. The overall relapse rate in our cohort was $10 \%$, of which three cases were related to noncompliance to medication, and of which one case had an earlier diagnosis of MSK brucellosis. Treatment-related complications were not reported in the study.

\section{CONCLUSION}

This study has demonstrated that MSK manifestation in brucellosis accounts for onethird of the cases. The index of suspicion should be high in brucellosis-endemic countries. Clinical symptoms, risk factors, specific laboratory findings, and clinical images are important for early recognition and treatment.

\section{References}

1. Godfroid J et al. From the discovery of the Malta fever's agent to the discovery of a marine mammal reservoir, brucellosis has continuously been a re-emerging zoonosis. Vet Res. 2005;36(3):313-26.

2. Pappas $\mathrm{G}$ et al. The new global map of human brucellosis. Lancet Infect Dis. 2006;6(2):91-9.

3. Al Shehhi $\mathrm{N}$ et al. Human brucellosis in the Emirate of Abu Dhabi, United Arab Emirates, 2010-2015. BMC Infect Dis. 2016;16(1):558.

4. Mohammed MA et al. Sero-prevalence and epidemiology of brucellosis in camels, sheep and goats in Abu Dhabi Emirate. Int J Anim Vet Adv. 2013;5(2):82-6.

5. Buzgan T et al. Clinical manifestations and complications in 1028 cases of brucellosis: a retrospective evaluation and review of the literature. Int $\mathrm{J}$ Infect Dis. 2010;14(6):e469-78.

6. Musallam II et al. Systematic review of brucellosis in the Middle East: disease frequency in ruminants and humans and risk factors for human infection.
Epidemiol Infect. 2016;144(4):671-85.

7. Dean AS et al. Clinical manifestations of human brucellosis: a systematic review and meta-analysis. PLoS Negl Trop Dis. 2012;6(12):e1929.

8. Alp E, Doganay M. Current therapeutic strategy in spinal brucellosis. Int J Infect Dis. 2008;12(6):573-7.

9. Chelli Bouaziz $M$ et al. Spinal brucellosis: a review. Skeletal Radiol 2008;37(9):785-90.

10. Pourbagher A et al. Epidemiologic, clinical, and imaging findings in Brucellosis patients with osteoarticular involvement. AJR Am J Roentgenol. 2006;187(4):873-80.

11. Sofian $M$ et al. Screening of family members of patients with acute brucellosis in an endemic area of Iran. Iran J Microbiol. 2013;5(3):215-9.

12. Ulu-Kilic A et al. Update on treatment options for spinal brucellosis. Clin Microbiol Infect. 2014;20(2):075-82.

13. Geyik MF et al. Musculoskeletal involvement of brucellosis in different age groups: a study of 195 cases. Swiss Med Wkly. 2002;132(7-8):98105.

14. Esmaeilnejad-Ganji SM, EsmaeilnejadGanji SMR. Osteoarticular manifestations of human brucellosis: a review. World J Orthop. 2019;10(2):54-62.

15. Bukharie HA. Clinical features, complications and treatment outcome of brucella infection: ten years' experience in an endemic area. Trop J Pharm Res. 2009;8(4):303-10.

16. Franco MP et al. Human brucellosis. Lancet Infect Dis. 2007;7(12):775-86.

17. Yousefi-Nooraie $R$ et al. Antibiotics for treating human brucellosis. Cochrane Database Syst Rev. 2012;10(10):CD007179.

18. Pappas $\mathrm{G}$ et al. Treatment of brucella spondylitis: lessons from an impossible meta-analysis and initial report of efficacy of a fluoroquinolone-containing regimen. Int J Antimicrob Agents. 2004;24(5):502-7. 